\title{
Influence of thermal and flow conditions on the temperature distribution in the evaporator tubes
}

\author{
Marek Majdak \\ Department of Energy, Cracow University of Technology, al. Jana Pawla II 37, 31-864 Cracow, Poland
}

\begin{abstract}
The article presents the results of thermal and flow analysis of the working conditions of neighbouring waterwall tubes, loaded with heat streams of different values. The numerical model used for the analysis, allowing to calculate the temperature distribution of the tubes and the fluid flowing through them at each time step depending on the thermophysical parameters of the fluid and the material from which the tubes were made. By using the algorithm it is possible to precisely determine the temperature distribution for tubes, allowing to determine the places where the most divergent temperatures occur and in which thermal stresses of the highest value may occur. Analysis for several adjacent tubes will allow for the effect of temperature differences in the tubes to the temperature of the fin which is connecting them and to collect data that may be used for the determination of stress distribution in the tubes and fins.
\end{abstract}

\section{Introduction}

In the combustion chambers of supercritical boilers there are thermal conditions that change significantly on the width of the furnace. The heat flux falling on the waterwall tubes installed in the furnace varies from minimum values in the corners of the chamber to maximum in the middle of the wall. The situation is additionally complicated by the fact that the heat flux falling on a single tube changes significantly also at the height of the furnace chamber $[1,2]$.

The parallel occurrence of these factors results in thermal stresses with high values that may result in damage to the tubes and the fins that connect them.

The author of the article have developed an algorithm that allows determining the distribution of temperatures in neighboring waterwall tubes taking into account the variability of the heat flux described above.

\section{Problem analysis}

In the combustion chambers of supercritical boilers there are thermal conditions that change significantly on the width of the furnace. Due to the need to ensure the flexibility of the block systems, the mass flow of the fluid flowing through the individual waterwall tubes also changes significantly.

The article presents an analysis of the temperature distribution in two neighbouring smooth waterwall tubes, which are mounted on the furnace wall of a supercritical steam boiler. Fluid parameters were determined in accordance with the assumptions presented in [3-5]. In order to increase the precision of calculations, were assumed that the waterwall tube has been divided into small control volumes.
The used model allows, among others, to determine fluids enthalpies, pressure distribution and mass flows in relation to the height above the inlet to the tube. Parameters of the working fluid have been determined using the International Association for the Properties of Water and Steam (IAPWS IF97) parameters table [6].

Equations known from the definition of these parameters (1-3) can be simplified and written as equations with separated variables.

$$
\begin{gathered}
\frac{\partial \dot{m}}{\partial z}=-A \frac{\partial p}{\partial \tau} \\
\frac{\partial}{\partial z}\left(\frac{m^{2}}{A^{2} \rho}+p\right)=-\frac{1}{A} \frac{\partial \dot{m}}{\partial \tau}-\frac{\partial p_{t}}{\partial z}-\rho g \sin \beta \\
\frac{\partial h}{\partial z}=\frac{\rho A}{\dot{m}}\left(-\frac{\partial h}{\partial \tau}+\frac{4 \alpha(\theta-t)}{d_{w} \rho}\right)
\end{gathered}
$$

After transformations on the left side of the equal sign are spatial derivatives. The time derivatives on the right side of the equations can be replaced with backward difference quotients.

In addition, in the case of the proposed method, the Courant condition must be met, according to which the speed at which the numerical solution moves $(\Delta z / \Delta \tau)$ must be greater than the physical speed (w):

$$
\Delta \tau \leq \frac{\Delta z}{w}
$$

In order to determine the temperature distribution in the tubes connected to the fins, there was developed the method of division the cross-section of the tube into control volumes based on the method presented in [7-9]. The tube cross-section was divided into control volumes, in which 36 characteristic points were identified (Fig. 1):

* Corresponding author: marek.majdak@mech.pk.edu.pl 


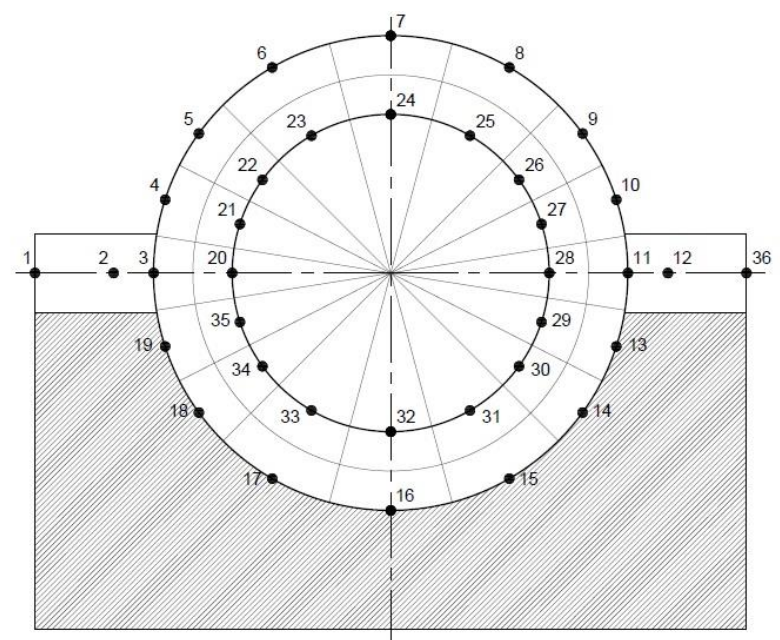

Fig. 1. Division of the cross-section of the waterwall tube with fins into control volumes

A sample equation to determine the temperature distribution, in this case for point 7 , can be written as follows:

$\theta_{7, j}^{\tau+\Delta \tau}=\theta_{7, j}^{\tau}$

$+\frac{\Delta \tau}{c_{7, j}^{\tau} \rho_{7, j}^{\tau} \frac{\Delta \hat{\varphi}_{1}}{2}\left(r_{o}^{2}-r_{m}^{2}\right) \Delta z}\left[\lambda_{7, j}^{\tau} \frac{\theta_{6, j}^{\tau}-\theta_{7, j}^{\tau}}{\Delta \widehat{\varphi}_{1} r_{o}} \frac{\Delta r}{2} \Delta z\right.$

$+\lambda_{7, j}^{\tau} \frac{\theta_{8, j}^{\tau}-\theta_{7, j}^{\tau}}{\Delta \hat{\varphi}_{1} r_{o}} \frac{\Delta r}{2} \Delta z+\lambda_{7, j}^{\tau} \frac{\theta_{24, j}^{\tau}-\theta_{7, j}^{\tau}}{\Delta r} \Delta \widehat{\varphi}_{1} r_{m} \Delta z$

$+\lambda_{7, j}^{\tau} \frac{\Delta \hat{\varphi}_{1}}{2}\left(r_{o}^{2}-r_{m}^{2}\right) \frac{\theta_{7, j-1}^{\tau}-\theta_{7, j}^{\tau}}{\Delta z}$

$\left.+\lambda_{7, j}^{\tau} \frac{\Delta \widehat{\varphi}_{1}}{2}\left(r_{o}^{2}-r_{m}^{2}\right) \frac{\theta_{7, j+1}^{\tau}-\theta_{7, j}^{\tau}}{\Delta z}+\dot{q}_{7, j}^{\tau} \Delta \widehat{\varphi}_{1} r_{o} \Delta z\right]$

In subscripts in Eq. (5) written in the form "X, $\mathrm{j}$ " " $\mathrm{X}$ " denotes the point number on the tube cross-section (Fig. 1) and subscript "j" varies in the range of $2, \ldots, \mathrm{M}$ and signifies the number of analysed cross-sections. Equations for the remaining are created analogously, based on geometrical relationships between nodes.

The parameters of waterwall tubes from one of the Polish power plants were selected as input data for the simulation. The lower part of furnace have approximately $32 \mathrm{~m}$ height and is made of $16 \mathrm{Mo} 3$ steel. The tubes in the lower part of the furnace have outer diameter $33.7 \mathrm{~mm}$ and wall $6.1 \mathrm{~mm}$, are spaced with a pitch $50 \mathrm{~mm}$ and set at an angle $24.62^{\circ}$. The tubes in the upper part are located above the pulverized fuel burners and are made of $13 \mathrm{CrMo} 4-5$ steel - material which can operate at higher temperatures. They have larger outer diameter $38.0 \mathrm{~mm}$, wall thickness $6.3 \mathrm{~mm}$ and pitch $57 \mathrm{~mm}$ and are set at a greater angle of inclination $28.36^{\circ}$. The analysed system corresponds to a fragment of the furnace wall of a boiler producing steam at a pressure of $26.6 \mathrm{MPa}$, temperature $554{ }^{\circ} \mathrm{C}$, and nominal power output capacity $858 \mathrm{MW}$.

\section{Results and discussion}

In order to generate a temperature distribution of a large diversity, has chosen a case where different heat flux incident to the surface of the tubes and the mass flow of the medium flowing into each tube is the same.

To ensure the transparency of the temperature distributions shown below (Fig. 2-5), a system consisting of two tubes was selected. A heat flux of $120 \%$ of the nominal value falls on the left tube, and a heat flux of $80 \%$ of the nominal value falls on the right tube.

Below, in Figures 2-5, the temperature distributions expressed in degrees Celsius, are shown in two tubes at four selected distances from the inlet to the waterwall tube: $40 \mathrm{~m}$ (Fig. 2), $80 \mathrm{~m}$ (Fig. 3), $120 \mathrm{~m}$ (Fig. 4) and $166 \mathrm{~m}$ (Fig. 5).

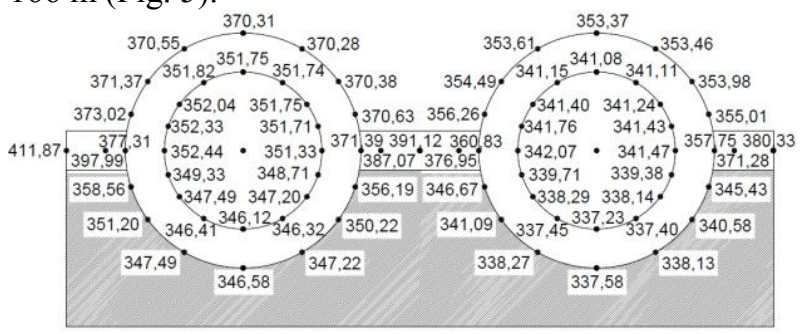

Fig. 2. Temperature distribution in two waterwall tubes at $40 \mathrm{~m}$ from the inlet (all temperatures are given in degrees Celsius)

As can be seen in the temperature distribution obtained above (in Fig. 2), in the middle of the fin connecting tubes is obtained temperature of above 390 degrees Celsius, on the face of the tubes in the range 350-370 degrees Celsius, and the temperature changes slightly on the rear wall of the tube and is in the range of 337-347 degrees Celsius.

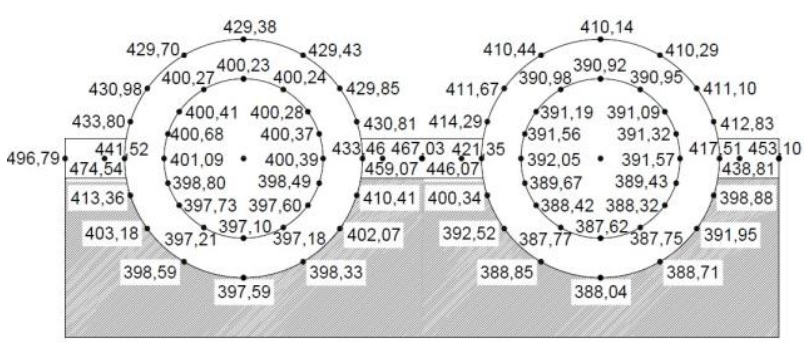

Fig. 3. Temperature distribution in two waterwall tubes at $80 \mathrm{~m}$ from the inlet (all temperatures are given in degrees Celsius)

In the temperature distribution obtained above (in Fig. 3), in the middle of the fin connecting tubes is obtained temperature of 467 degrees Celsius, on the face of the tubes in the range 410-430 degrees Celsius, the temperature on the rear wall of the tube is in the range of 388-398 degrees Celsius. 


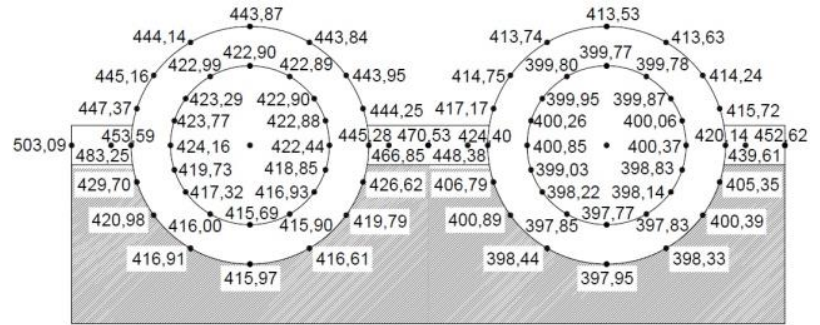

Fig. 4. Temperature distribution in waterwall tubes at $120 \mathrm{~m}$ from the inlet (all temperatures are given in degrees Celsius)

As can be seen in the temperature distribution obtained above (in Fig. 4), in the middle of the fin connecting tubes is obtained temperature of 470 degrees Celsius, on the face of the tubes in the range 413-443 degrees Celsius, and the temperature on the rear wall of the tube is in the range of 398-416 degrees Celsius.

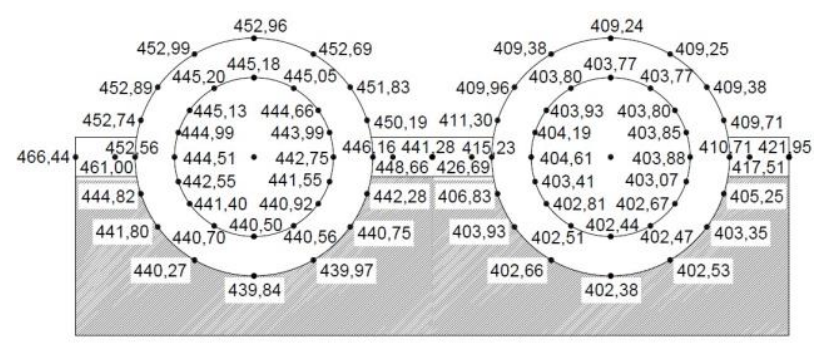

Fig. 5. Temperature distribution in waterwall tubes at $166 \mathrm{~m}$ from the inlet (all temperatures are given in degrees Celsius)

In the temperature distribution presented in Fig. 5, in the middle of the fin connecting tubes is obtained temperature of 441 degrees Celsius, on the face of the tubes in the range 409-453 degrees Celsius, and the temperature on the rear wall of the tube is in the range of 402-440 degrees Celsius.

\section{Conclusions}

The temperature distributions presented above indicate a very large variety of temperatures occurring in waterwall tubes loaded with heat streams of different values. At the highest analysed heat load values, the temperature differences between the front and rear surfaces on the same cross-section of a single tube exceed 30 degrees Celsius. In turn, the maximum temperature difference obtained at the level of $120 \mathrm{~m}$ from the inlet to the tube (Figure 5) is over 40 degrees Celsius. Due to the uneven heat load falling on the tubes in the vertical, there is also a very large variation in the temperature changes of the tubes and the fins that connect them. In the area in which the heat flux falling on the waterwall tubes begins to decrease, fins connecting the tubes rapidly cool down, and the rear walls of tubes increase their temperature because they are heated by the high-temperature medium flowing through them.

The occurrence of such diverse metal temperatures at a small distance from one another influences the occurrence of high thermal stresses. The author plans to conduct further analyses aimed at creating a model that allows determining the distribution of thermal stresses in the analysed system. After generating the stress distribution, analyses will be carried out to determine changes in the design of combustion chamber walls that will help in reducing thermal stresses, and consequently will prevent more damage resulting from overheating of elements or exceeding the permissible stress value.

\section{References}

1. M. Majdak, S. Gradziel, Therm. Sci. 24 (supp. 4), 1333-1344 (2019)

2. M. Majdak, S. Grądziel, MATEC Web Conf. 240, 1-4 (2018)

3. S. Grądziel, K. Majewski, Heat Transfer Eng. 39, 1243-1250 (2017)

4. S. Grądziel, Energy 172, 881-891 (2019)

5. S. Grądziel, K. Majewski, Chem. Process Eng. 37 (2), 199-213 (2016)

6. W. Wagner, et al., J. Eng. Gas Turb. Power 122, 150-182 (2000)

7. W. Zima, M. Nowak-Oclon, Procedia Eng. 157, 200-206 (2016)

8. W. Zima, M. Nowak-Oclon, Heat Transfer Eng. 39, 1272-1282 (2017)

9. W. Zima, et al., Energy 148, 809-823 (2018) 